\title{
Functional Movement Is Negatively Associated with Weight Status and Positively Associated with Physical Activity in British Primary School Children
}

\author{
Michael J. Duncan and Michelle Stanley \\ Department of Biomolecular and Sports Sciences, Coventry University, Coventry CV1 5FB, UK \\ Correspondence should be addressed to Michael J. Duncan, aa8396@coventry.ac.uk
}

Received 29 November 2011; Revised 17 January 2012; Accepted 21 January 2012

Academic Editor: George P. Nassis

Copyright ( 2012 M. J. Duncan and M. Stanley. This is an open access article distributed under the Creative Commons Attribution License, which permits unrestricted use, distribution, and reproduction in any medium, provided the original work is properly cited.

\begin{abstract}
Although prior studies have suggested that overweight and obesity in childhood are associated with poorer functional movement performance, no study appears to have examined this issue in a pediatric population. The relations between BMI, ambulatory physical activity and functional movement screen (FMS) performance were compared in 58, 10-11-year-old children. Total FMS score was significantly, negatively correlated with BMI $(P=.0001)$ and positively related to PA $(P=.029)$. Normal weight children scored significantly better for total FMS score compared to children classified as overweight/obese $(P=.0001)$. Mean \pm S.D. of FMS scores were $15.5 \pm 2.2$ and $10.6 \pm 2.1$ in normal weight and overweight/obese children, respectively. BMI and PA were also significant predictors of functional movement $\left(P=.0001\right.$, Adjusted $\left.R^{2}=.602\right)$ with BMI and PA predicting $52.9 \%$ and $7.3 \%$ of the variance in total FMS score, respectively. The results of this study highlight that ambulatory physical activity and weight status are significant predictors of functional movement in British children. Scientists and practitioners therefore need to consider interventions which develop functional movement skills alongside physical activity and weight management strategies in children in order to reduce the risks of orthopaedic abnormality arising from suboptimal movement patterns in later life.
\end{abstract}

\section{Introduction}

Overweight and obesity in childhood are recognised as a major health problem worldwide [1] and while considerable data have been published relating to influences such as sedentary behaviour and nutritional habits on weight status, there is a dearth of information pertaining to the structural and functional limitations of excess weight in adults [2,3], with even less data in children. This is despite authors noting that children display alterations to their functional movement as a consequence of excessive weight [2] and suboptimal movement patterns found in overweight and obesity can seriously impede daily physical activity level and limit functional performance $[3,4]$. The concern here is that an inability to complete fundamental daily tasks coupled with excessive and prolonged loading of tissues in childhood may lead to orthopaedic abnormality in later life. Therefore, minimizing joint deterioration from excessive joint loading or impaired movement patterns evident in obese and overweight children should be treated at the earliest opportunity [5]. However, no study appears to have examined these associations in children. Therefore, the aim of this study was to examine relations between habitual physical activity, functional movement patterns, and weight status in children.

\section{Materials and Methods}

2.1. Participants. Following institutional ethics approval, fifty-eight children (29 boys and 29 girls, 86\% Caucasian) from a primary school in Central England volunteered and returned signed parental informed consent forms to participate in the study. Children were from school year 6 (aged $10-11$ years) and were predominantly Caucasian (81\%). The mean age (SD) of the children was $10.7(0.4)$ years. The school the children was drawn from was in an urban area 


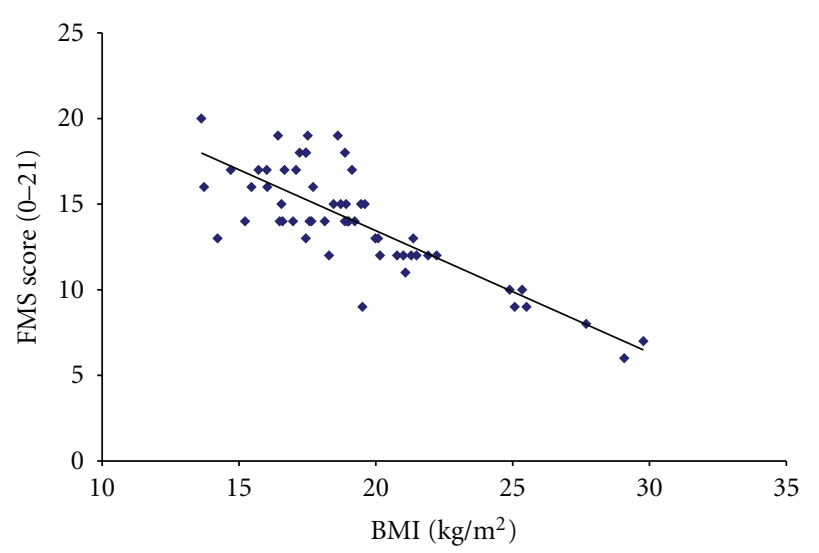

(a)

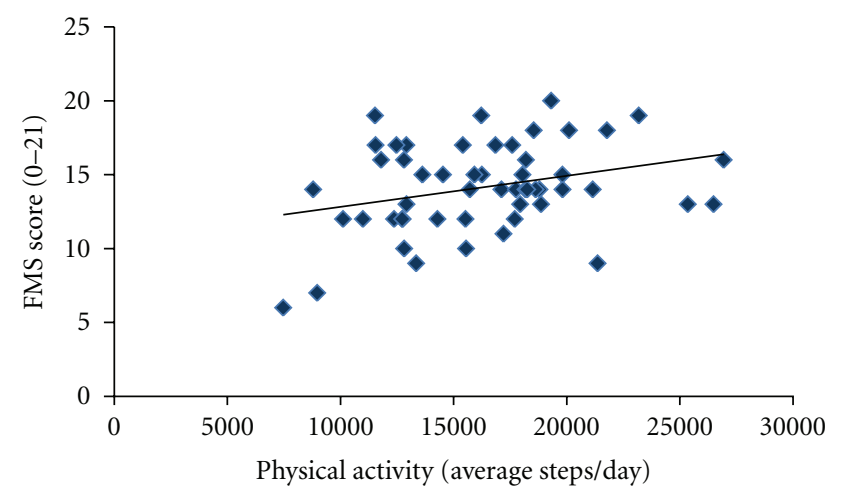

(b)

FIGURE 1: Scattergraph illustrating the relationship between; (a) FMS score and BMI and (b) FMS score and physical activity in British children.

of the city of Coventry and was located in the least deprived ward in the city with an average household income of

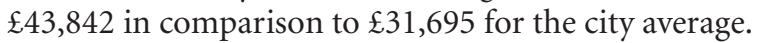

\subsection{Procedures}

2.2.1. Anthropometry. Body mass ( $\mathrm{kg})$ and height $(\mathrm{m})$ were measured using a Stadiometer and weighing scales (Seca Instruments, Germany, Ltd) prior to the monitoring period. From this, body mass index was determined as $\mathrm{kg} / \mathrm{m}^{2}$. Children were classified as normal weight (68.4\%) and overweight/obese (31.6\%) according to IOTF criteria [6].

2.2.2. Physical Activity Assessment. Physical activity was assessed using a sealed, piezoelectric pedometer (New Lifestyles, NL2000, Montana, USA) worn over four days (2 $\times$ weekdays and $2 \times$ weekend days) in accordance with recommendations for the assessment of physical activity in children [7] and using protocols previously described [1]. Across the measurement period, the children completed a brief survey to verify that the pedometers were worn for the entire time of the study. Survey results were used to identify participants who reported removing the pedometer for $\geq 1 \mathrm{~h}$, resulting in three exclusions from the data set, similar to other studies [8].

2.2.3. Functional Movement Assessment. The Functional Movement Screen (FMS) is a preparticipation screening tool which evaluates the fundamental movement patterns that underpin performance of all movement $[9,10]$. The FMS consists of seven tests which challenge an individual's ability to perform basic movement patterns that reflect combinations of muscle strength, flexibility, range of motion, coordination, balance, and proprioception $[9,10]$. The FMS was administered by a trained rater using standardised procedures, instructions, and scoring processes $[9,10]$. Each participant was given 3 trials on each of the seven tests (deep squat, hurdle step, in-line lunge, shoulder mobility, active straight leg raise, trunk-stability push-up, and rotary stability) in accordance with recommended guidelines [9, 10]. Each trial was scored from 0 to 3 with higher scores reflecting better functional movement. For each test, the highest score from the three trials was recorded and used to generate an overall composite FMS score with a maximum value of 21 and in accordance with recommended protocols $[9,11]$.

2.3. Data Analysis. Relationships between total FMS score, BMI and PA (average steps/day) were analysed using Pearson's product moment correlations. A 2 (Gender) $\times 2$ (weight status: normal weight versus overweight/obese) $\times$ 2 (PA: children meeting/not meeting the health related steps/day cut off (8)) analysis of variance (ANOVA), using backwards elimination to achieve a parsimonious solution, was employed to examine any differences in functional movement (FMS score) according to gender, weight status, and physical activity. Where any significant differences were detected, Bonferroni post-hoc multiple comparisons were used to detect where these differences lay. Multiple linear regression was also employed to predict functional movement (FMS score) from BMI and Physical activity. Statistical significance was set a priori as $P=.05$.

\section{Results}

Total FMS score was significantly, negatively correlated with BMI $(r=-.806, P=.0001$, See Figure $1(\mathrm{a}))$ and positively related to $\mathrm{PA}(r=.301, P=.029$, see Figure $1(\mathrm{~b}))$. Furthermore, ANOVA found a significant main effect for weight status (F 1, $53=50.4, P=.0001$ ) for total FMS score with normal weight children scoring significantly better than their overweight and obese peers (Mean Diff $=4.941, P=.0001$, see Figure 2$)$. Mean \pm S.D. of FMS scores were $15.5 \pm 2.2$ and $10.6 \pm 2.1$ in normal weight and overweight/obese children, respectively. Multiple linear regression also revealed a significant predictor model (F 2, $53=40.369, P=.0001$, Adjusted $R^{2}=.602$ ) whereby BMI and physical activity (average steps/day) predicted $60.2 \%$ 
TABLE 1: Mean \pm S.D. of total FMS score, physical activity (average steps/day), and BMI between gender and weight status groups.

\begin{tabular}{lcccccr}
\hline & \multicolumn{2}{c}{ Total FMS score } & \multicolumn{2}{c}{$\begin{array}{c}\text { Physical activity } \\
\text { (Avg steps/day) }\end{array}$} & \multicolumn{2}{c}{$\begin{array}{c}\text { BMI } \\
\left(\mathrm{kg} / \mathrm{m}^{2}\right)\end{array}$} \\
& Mean & S.D. & Mean & S.D. & Mean & S.D. \\
\hline Boys $(n=29)$ & 13.5 & 3.4 & 17480 & 4818 & 19.9 & 4.7 \\
Girls $(n=29)$ & 14.5 & 2.8 & 15259 & 3585 & 19.1 & 2.9 \\
Normal weight $(n=39)$ & 15.5 & 2.2 & 17078 & 4009 & 17.5 & 1.7 \\
Overweight/obese $(n=19)$ & 10.6 & 2.1 & 14522 & 4602 & 23.3 & 0.9 \\
\hline
\end{tabular}

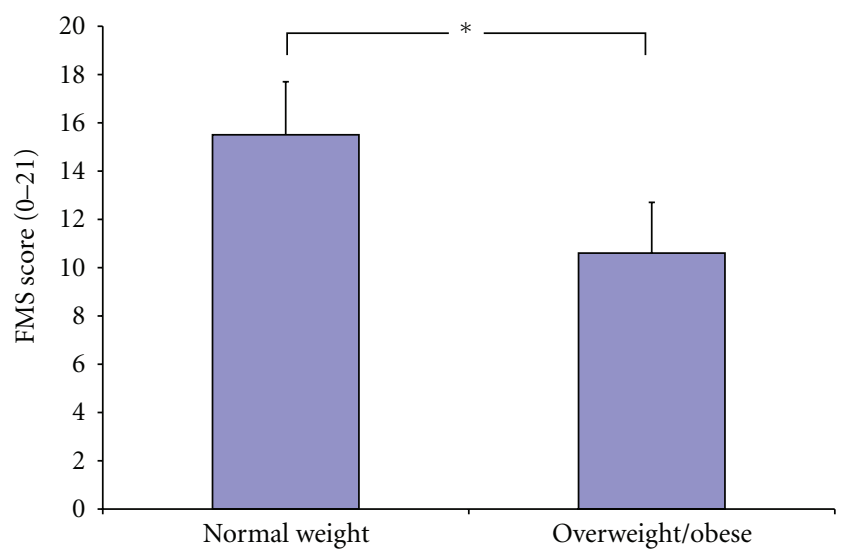

FIGURE 2: Mean \pm S.D. of total FMS score between children classified as normal weight and overweight/obese according to IOTF criteria $\left({ }^{*} P=.0001\right)$.

of the variance in functional movement score. However, BMI was a stronger predictor $(\beta=-.712, P=.0001)$ of functional movement predicting $52.9 \%$ of the variance in total FMS score in comparison to physical activity $(\beta=$ $1200.0, P=.03$ ) where average steps/day predicted $7.3 \%$ of the variance in FMS score. Mean \pm S.D. of total FMS score, PA (average steps/day), and BMI between gender and weight status groups are presented in Table 1 .

\section{Discussion}

Results of this study suggest that functional movement is related to weight status and habitual physical activity, with weight status having a stronger association with functional movement in this population. In this case although BMI and physical activity were both significant predictors of functional movement predicting $60.2 \%$ of the variance in FMS score, BMI alone predicted $52.9 \%$ of this variance in comparison to physical activity, where average steps/day predicted $7.3 \%$ of the variance in FMS score. This study supports prior research in adults which has suggested that excess weight results in functional limitations $[2,3]$ and assertions that children display altered functional movement as a consequence of excess weight [2]. The positive association seen between FMS score and habitual physical activity in the present study supports prior assertions that the lack of physical activity is associated with the lack of functional movement skills in adults [4]. However, in the context of the present study and population examined, it is possible that functional limitation may have existed prior to overweight/obesity. As such, excess weight and/or functional prowess are the result of natural selection since children who are functionally limited will remain inactive and will not develop the fundamental movement patterns that underpin performance to the same level of mastery as children who do not have a functional limitation. In the same way, children who are not functionally limited may more likely enjoy physical activity and thus, engage in more regular practise of the fundamental movement patterns that underpin performance. These children will consequently become more proficient in performance of fundamental movement patterns resulting in greater physical self efficacy and increased likelihood of participation in physical activity. To the authors knowledge this is the first study to examine associations between weight status, physical activity, and FMS in a pediatric population. The data presented here are however, important as they highlight those children who are overweight/obese present maladaptive movement patterns needed to accomplish tasks of daily life. Over time, these movement patterns coupled with the effect of excess weight on joint loading are likely to lead to orthopaedic abnormality in later life [4]. Moreover, such suboptimal movement patterns can prevent individuals from undertaking health enhancing physical activity [4].

There are several proposed mechanisms for the impact of overweight and obesity on functional movement. However, these suggestions have tended to be based on studies in adult populations with few studies examining this issue in children and adolescents. One suggestion is that overweight and obesity lead to alterations in the musculoskeletal system that place overweight individuals at higher risk of musculoskeletal pain $[4,12]$. Such pain may then result in restricted range of movement and/or lower levels of physical activity. One study by Messier et al. [12] showed that a weight loss of approximately $5 \%$ following a combined diet and exercise programme improved physical function, mobility, and reduced pain in overweight and obese adults with osteoarthritis. However, musculoskeletal pain is considered a chronic condition that is less likely to affect children. Some studies in children and adolescents $[13,14]$ have evidenced changes in foot structure and plantar pressure distribution, as a result of structural adaptation to excess weight, which was suggested to lead to functional movement complications. Likewise, research by Zoico et al. [15], with older adults, has 
concluded that a BMI of $30 \mathrm{~kg} \mathrm{~m}^{2}$ or greater is significantly associated with functional limitation. However, Stenholm et al. [16] has further suggested that it is the ratio of fat free mass (FFM) to body mass which is important as individuals with higher FFM are more likely to be functionally proficient than those with lower FFM. However, while these studies were conducted with older adults, there is a lack of data examining the association between BMI, FFM, muscle strength, and functional movement in children [4]. Future research would therefore be welcomed in this area.

Similarly, only one other study to date [17] has reported specifically on the use of the Functional Movement Screen in a pediatric population. Burton et al. [17] reported that, in a sample of 39 middle school children, there were significantly higher total FMS scores in girls compared to boys, principally due to better performance in the deep squat, in line lunge, straight leg raise, and shoulder rotation. They concluded that girls exhibit superior quality in functional movement compared to boys but suggested that it was important to develop musculoskeletal interventions to improve functional movement in children and adolescents. However, although Burton et al. [17] did not account or control for variation in weight status in their study making it difficult to draw parallels with the current study, the range of total FMS scores reported in their study [17] is similar to those documented in this study. However, there was no gender difference found for FMS score in the present study but as Burton et al. [17] did not assess or control for weight status it is difficult to draw parallels between the two studies.

Irrespective of whether it is functional limitation that results in lack of physical activity and increases in overweight/obesity or whether lack of physical activity and overweight/obesity leads to suboptimal movement, the data present here support the need for interventions to increase habitual physical activity and improve functional movement in British children generally but those who are overweight and obese specifically. It is also important to note that there may be other factors which influence performance of the functional movement screen in children such as motivation to perform the movement patterns and particularly motor learning. Although the children in the present study had been familiarized with the movements involved in the FMS, it may be that there are practice effects in the FMS. No studies to date have examined this issue and the recommended protocols were followed when administering the FMS into the current study. However, future research examining the effects of motor learning on performance of the FMS would be useful in elucidating this area further.

In respect to the clinical significance of the current study, the results present here do not necessarily suggest a clinical need for those children exhibiting poor functional movement at their present age. The worry, however, is that if suboptimal movement patterns, present in children who are overweight/obese, persist into adolescence and adulthood, this will lead to a range of musculoskeletal problems of clinical significance. This includes a range of factors including knee osteoarthritis, early need for hip replacement and chronic pain, in addition to limitations in tasks of daily living (see [4] for a review). Thus, in overweight/obese children who exhibit suboptimal functional movement, interventions aimed at improving the quality of functional movement via weight loss and/or increases in physical activity energy expenditure may offset more severe clinical implications in later life.

Considering the association between physical activity and FMS found in this study, it may be that the relationship between physical activity and weight status is magnified as children become older if their weight is not managed, resulting in further reduction in physical activity, functional movement, or both. This suggestion is, however, speculative and further research is needed to verify this claim. This exploratory study is also limited by a small sample size and larger scale studies would be welcomed to verify the claims made here. In addition, cause and effect in relation to physical activity and functional movement could not be determined in the present sample.

\section{Conclusion}

The results of this study do, however, highlight that ambulatory physical activity and weight status are significant predictors of functional movement in British children. In the current study weight status had a greater influence on functional movement compared to physical activity and children who were classified as overweight/obese demonstrated significantly poorer functional movement than children classified as normal weight.

\section{Conflict of Interests}

The authors declare they have no conflict of interests.

\section{References}

[1] M. J. Duncan, A. Nevill, L. Woodfield, and Y. Al-Nakeeb, “The relationship between pedometer-determined physical activity, body mass index and lean body mass index in children," International Journal of Pediatric Obesity, vol. 5, no. 5, pp. 445450, 2010.

[2] A. P. Hills, E. M. Hennig, N. M. Byrne, and J. R. Steele, "The biomechanics of adiposity-structural and functional limitations of obesity and implications for movement," Obesity Reviews, vol. 3, no. 1, pp. 35-43, 2002.

[3] S. C. Wearing, E. M. Hennig, N. M. Byrne, J. R. Steele, and A. P. Hills, "The biomechanics of restricted movement in adult obesity," Obesity Reviews, vol. 7, no. 1, pp. 13-24, 2006.

[4] J. Nantel, M.-E. Mathieu, and F. Prince, "Physical activity and obesity: biomechancial and physiological key concepts," Journal of Obesity, vol. 2011, Article ID 650230, 10 pages, 2011.

[5] R. H. Rozendal, "Biomechanics of standing and walking," in Disorders of Gait and Posture, W. Bles and T. Brandt, Eds., pp. 3-18, Elsevier Scientific, Amsterdam, The Netherlands, 1986.

[6] T. J. Cole, M. C. Bellizzi, K. M. Flegal, and W. H. Dietz, "Establishing a standard definition for child overweight and obesity worldwide: international survey," British Medical Journal, vol. 320, no. 7244, pp. 1240-1243, 2000.

[7] S. G. Trost, "Objective measurement of physical activity in youth: current issues, future directions," Exercise and Sport Sciences Reviews, vol. 29, no. 1, pp. 32-36, 2001. 
[8] C. Tudor-Locke, R. P. Pangrazi, C. B. Corbin et al., "BMIreferenced standards for recommended pedometer-determined steps/day in children," Preventive Medicine, vol. 38, no. 6, pp. 857-864, 2004.

[9] G. Cook, L. Burton, and B. Hoogenboom, "Pre-participation screening: the use of fundamental movements as an assessment of function-part 1," North American Journal of Sports Physical Therapy, vol. 1, pp. 62-72, 2006.

[10] G. Cook, L. Burton, and B. Hoogenboom, "Pre-participation screening: the use of fundamental movements as an assessment of function-part 2," North American Journal of Sports Physical Therapy, vol. 1, pp. 132-139, 2006.

[11] A. G. Schneiders, A. Davidsson, E. Horman, and J. S. Sullivan, "Functional Movement Screen Normative values in a young active population," International Journal of Sports Physical Therapy, vol. 6, pp. 75-82, 2011.

[12] S. P. Messier, R. F. Loeser, M. N. Mitchell et al., "Exercise and weight loss in obese older adults with knee osteoarthritis: a preliminary study," Journal of the American Geriatrics Society, vol. 48, no. 9, pp. 1062-1072, 2000.

[13] D. L. Riddiford-Harland, J. R. Steele, and L. H. Storlien, "Does obesity influence foot structure in prepubescent children?" International Journal of Obesity, vol. 24, no. 5, pp. 541-544, 2000.

[14] A. M. Dowling, J. R. Steele, and L. A. Baur, "What are the effects of obesity in children on plantar pressure distributions?" International Journal of Obesity, vol. 28, no. 11, pp. 1514-1519, 2004.

[15] E. Zoico, V. Di Francesco, J. M. Guralnik et al., "Physical disability and muscular strength in relation to obesity and different body composition indexes in a sample of healthy elderly women," International Journal of Obesity, vol. 28, no. 2, pp. 234-241, 2004.

[16] S. Stenholm, D. Alley, S. Bandinelli et al., "The effect of obesity combined with low muscle strength on decline in mobility in older persons: results from the InCHIANTI Study," International Journal of Obesity, vol. 33, no. 6, pp. 635-644, 2009.

[17] R. Burton, K. Elkins, K. B. Kiesel, and P. J. Plisky, “Gender differences in functional movement screen and Y-balance test scores in middle aged school children," Medicine \& Science in Sports \& Exercise, vol. 41, p. 183, 2009. 


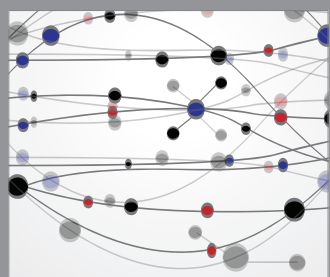

The Scientific World Journal
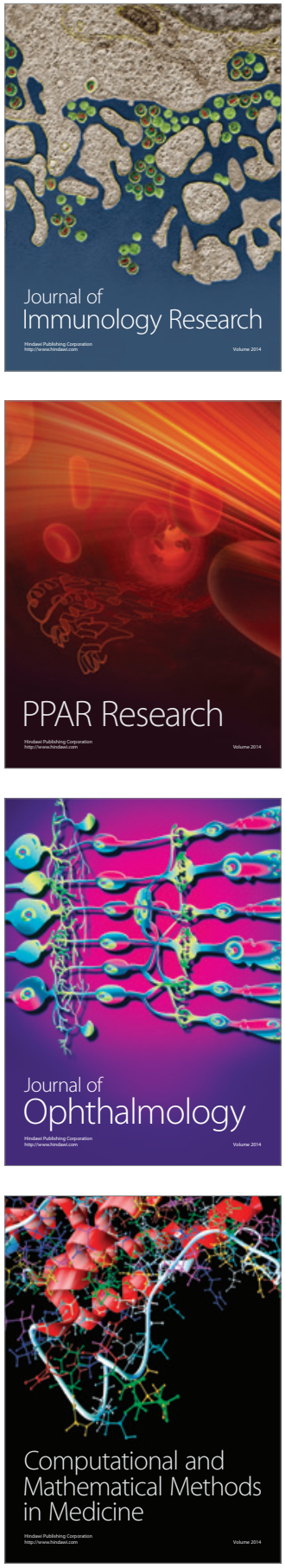

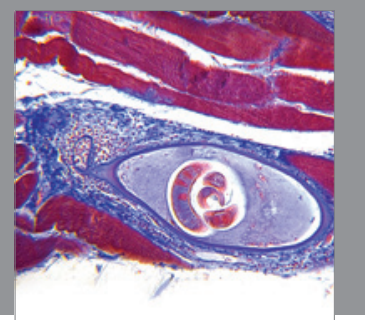

Gastroenterology

Research and Practice
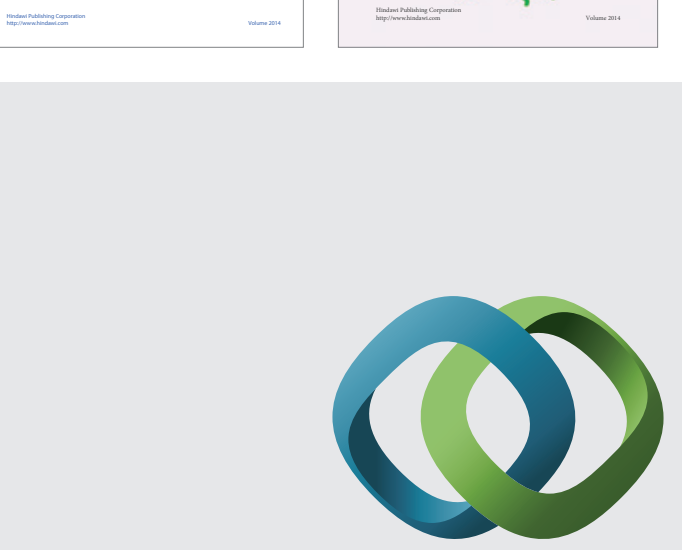

\section{Hindawi}

Submit your manuscripts at

http://www.hindawi.com
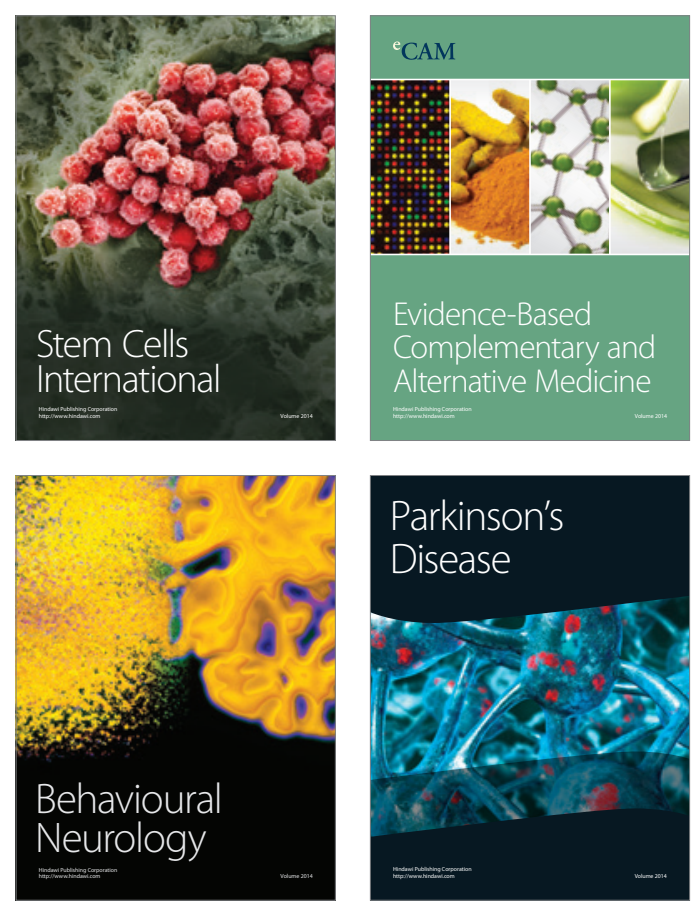

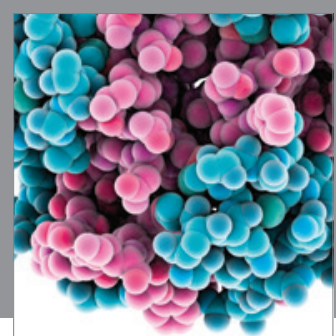

Journal of
Diabetes Research

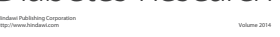

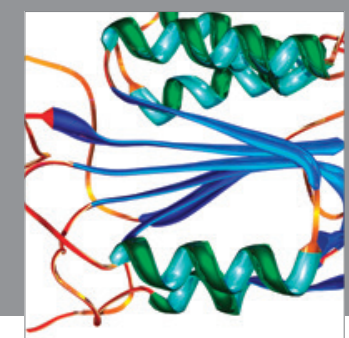

Disease Markers
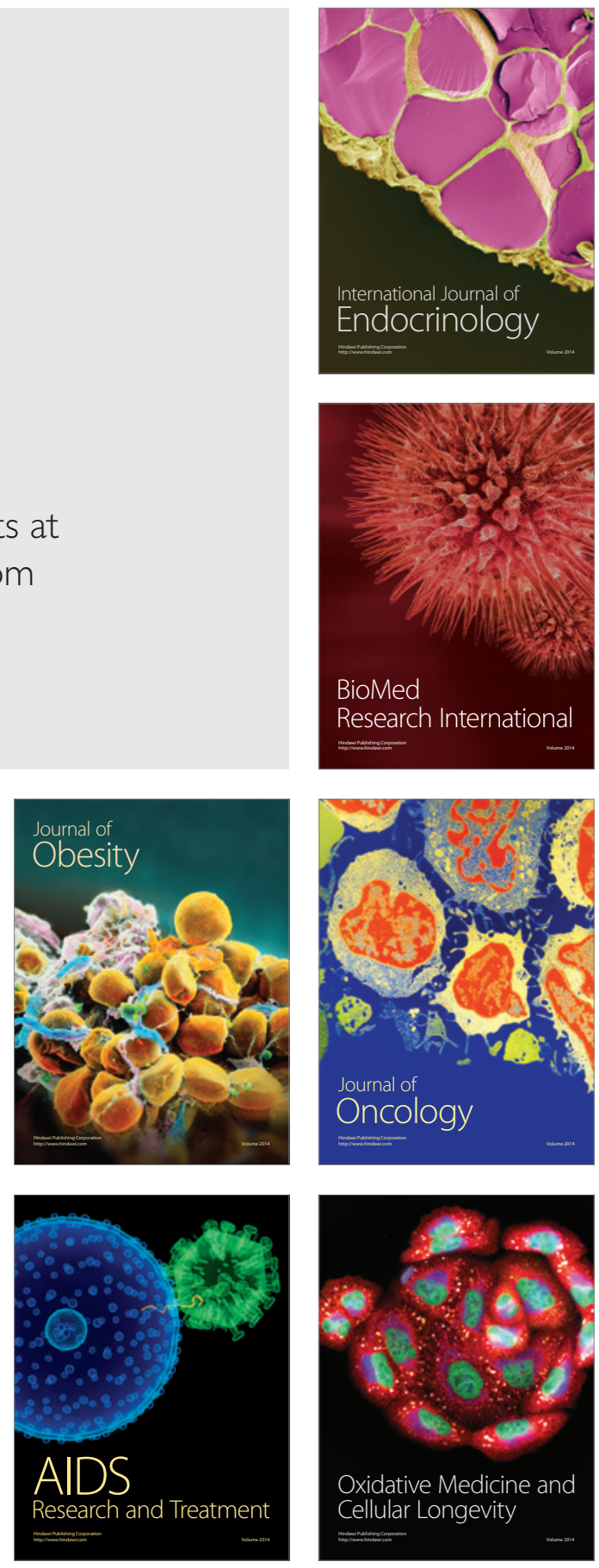